\title{
Usefulness of MIBG scintigraphy in idiopathic REM sleep behavior disorder: a systematic review
}

This article was published in the following Dove Press journal:

Research and Reports in Nuclear Medicine

24 January $201 \mathrm{I}$

Number of times this article has been viewed

Giorgio Treglia'

Fabrizio Cocciolillo'

Antonella Stefanelli'

Ernesto Cason ${ }^{2}$

Alessandro Giordano'

'Institute of Nuclear Medicine, Catholic University of the Sacred Heart, Rome, Italy; ${ }^{2}$ Unit of Nuclear Medicine, Maggiore Hospital, Bologna, Italy
Correspondence: Giorgio Treglia Institute of Nuclear Medicine, Catholic University of the Sacred Heart, Largo Gemelli, 8, Rome 00168, Italy

$\mathrm{Tel}+390630156200$

$\mathrm{Fax}+39063013745$

Email giorgiomednuc@libero.it
Background and purpose: It has been widely demonstrated that Parkinson's disease (PD) and dementia with Lewy bodies (DLB) share one clinicopathological entity; Lewy body disease (LBD) has thus become a general term for these diseases. LBD presents an impairment of adrenergic function and consequently an abnormal myocardial metaiodobenzylguanidine (MIBG) innervation imaging. Like PD and DLB, other Lewy body disorders, including pure autonomic failure and idiopathic rapid eye movement sleep behavior disorder (RBD), have been reported to develop cardiac sympathetic denervation. This study is designed to systematically review whether iodine-123-MIBG scintigraphy in patients with RBD demonstrates reduced myocardial uptake thereby suggesting cardiac sympathetic denervation, a typical finding of LBD, in these patients.

Methods: A comprehensive computer literature search of studies published through December 2010 regarding MIBG scintigraphy in patients with RBD was performed in PubMed/Medline and EMBASE databases. Only studies in which MIBG scintigraphy was performed in more than two patients with RBD were selected.

Results: Ultimately, six studies comprising a total of 139 patients with RBD were identified and discussed.

Conclusions: RBD usually show a markedly reduced cardiac MIBG uptake, consistent with a sympathetic denervation, a typical finding of a LBD.

Keywords: metaiodobenzylguanidine, REM sleep behavior disorder, Lewy body disease

\section{Introduction}

Radiolabeled metaiodobenzylguanidine (MIBG) is considered as an established sympathetic neuron imaging agent useful to study the organs richly innervated by the sympathetic nervous system. ${ }^{1-4}$

In recent years, it has been revealed that Parkinson's disease (PD) and dementia with Lewy bodies (DLB) share one clinicopathological entity: Lewy body disease (LBD) has thus become a general term for these diseases. ${ }^{5}$

LBD presents an impairment of adrenergic function and consequently an abnormal myocardial MIBG innervation imaging: the involvement of myocardial postganglionic sympathetic nerves may account for the reduction of myocardial MIBG uptake in patients with DLB, as well as in patients with PD. ${ }^{2-5}$

Like PD and DLB, other Lewy body disorders, including pure autonomic failure and idiopathic rapid eye movement (REM) sleep behavior disorder (RBD), have been reported to develop cardiac sympathetic denervation. Several MIBG studies have demonstrated that cardiac sympathetic denervation occurs in patients with LBD. ${ }^{2-4,6-8}$ submit your manuscript $\mid$ www.dovepress.com

Dovepress

DOI: 10.2147/RRNM.S16143
Research and Reports in Nuclear Medicine 201 I:I I-7

(C) 201 I Treglia et al, publisher and licensee Dove Medical Press Ltd. This is an Open Access article which permits unrestricted noncommercial use, provided the original work is properly cited. 
Recent evidence supports the notion that myocardial sympathetic involvement is a very early event in the evolution of LBD in humans, occurring prior to the deposition of $\alpha$-synuclein pathology in the central nervous system in many patients. ${ }^{9,10}$

Several investigators have, therefore, suggested that myocardial MIBG scintigraphy could be used to identify those with underlying LBD regardless of the other clinical features being exhibited; therefore, reduced myocardial MIBG uptake may be one of the most specific biomarkers for underlying LBD. ${ }^{10}$

RBD is a parasomnia manifested by vivid, often frightening dreams associated with simple or complex motor behavior during REM sleep. RBD is characterized by loss of normal skeletal muscle atonia during REM sleep with prominent motor activity and dreaming. The polysomnographic features of RBD include increased electromyographic tone with dream enactment behavior during REM sleep. ${ }^{10}$

The term idiopathic RBD refers to RBD occurring in the absence of any other obvious associated neurologic disorder. There is a large amount of recent data suggesting that many patients with idiopathic RBD actually represent an evolving neurodegenerative disorder. ${ }^{10}$

Over $50 \%$ of patients with idiopathic RBD will develop a synuclein-mediated neurodegenerative disorder, including PD, DLB, and multiple system atrophy (MSA). ${ }^{10-13}$

Braak and colleagues have proposed a staging system for the neuropathologic characterization of the phenotype of PD, and this system may be applicable to the timing of the evolution of RBD in the context of evolving LBD regardless of the clinical phenotype evolving as PD or DLB. ${ }^{9,10}$ This staging system posits a temporal sequence of $\alpha$-synuclein pathology in the brain, beginning mainly in the medulla (and olfactory bulb) and gradually ascending to more rostral structures. This temporal sequence of pathology could explain why RBD precedes parkinsonism, cognitive decline, and dementia in many patients with Lewy body pathology., 10

LBD is, therefore, a more systemic process that clearly affects the peripheral autonomic nervous system, and in some cases, actually may begin in the spinal cord prior to the brain and the peripheral autonomic system even before the central nervous system. ${ }^{9,10}$

The purpose of this study is to systematically review published data to evaluate whether MIBG scintigraphy in patients with RBD demonstrates reduced myocardial uptake thereby suggesting cardiac sympathetic denervation, a typical finding of $\mathrm{LBD}$, in these patients.

\section{Methods}

\section{Scintigraphic method}

MIBG is an analog of guanethidine and is taken up by the postganglionic presynaptic nerve endings of the adrenergic nervous system. After depolarization, MIBG is released into the synaptic cleft like norepinephrine, but is not metabolized. Labeling MIBG with iodine-123 permits the visualization of adrenergic innervation in vivo; MIBG scintigraphy not only displays the presence of noradrenergic innervation, but also its functional capability. ${ }^{1-4}$

About myocardial innervation imaging, radiolabeled MIBG is intravenously administered at rest, and early (from 10 to $30 \mathrm{~min}$ after injection) and delayed (from 3 to $4 \mathrm{~h}$ after injection) images are obtained (see Figures 1 and 2). Planar images with anterior view are adequate for the evaluation of cardiac sympathetic function. Tomographic images (single photon emission computed tomography) are often acquired to evaluate the three-dimensional myocardial uptake pattern. ${ }^{1-4}$

The most common semiquantitative indices used for interpretation of myocardial innervation images are the heart to mediastinum (H/M) ratio and the washout rate (WR) obtained from the anterior planar images. Regions of interest (ROIs) are set in the heart (H; target region) and the mediastinum (M; background region) in early and delayed images to obtain the mean count in each ROI, after which the H/M ratio is calculated. Based on the resulting ratio, the degree of uptake in the heart is evaluated. The WR is an index that indicates the rate at which MIBG is washed out between the early image and the delayed image, via comparison of cardiac counts in the early image. Normal values of these indices have been

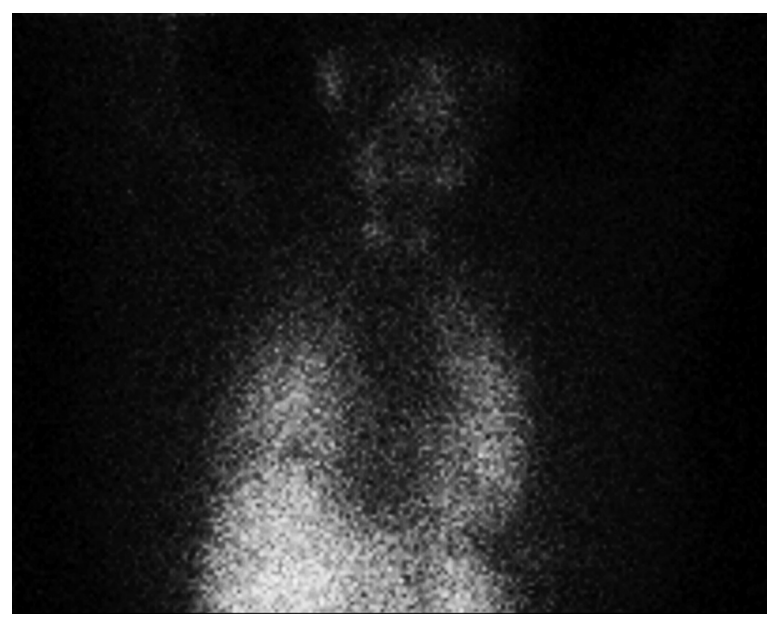

Figure I Normal uptake pattern of I-I23-MIBG scintigraphy (anterior view at $4 \mathrm{~h}$ after injection of III MBq); the tracer uptake in salivary glands, liver, heart, and lungs is considered as physiological. 


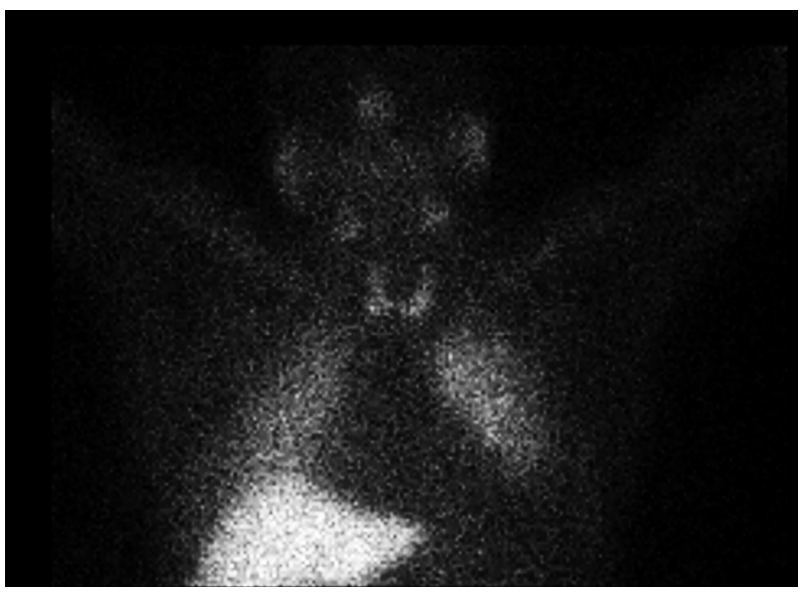

Figure 2 Abnormal uptake pattern of I-I23-MIBG scintigraphy (anterior view at $4 \mathrm{~h}$ after injection of I I I MBq) in a patient with RBD. No tracer uptake is observed in the heart suggesting a sympathetic denervation, a typical finding of Lewy body disease.

calculated performing MIBG scintigraphy in control patients and are different between various institutions depending on acquisition conditions. ${ }^{1-4}$

\section{Search strategy}

A comprehensive computer literature search of the PubMed/ MEDLINE and EMBASE databases was conducted to find relevant published articles on the usefulness of MIBG scintigraphy in patients with RBD. We used a search algorithm that was based on a combination of the terms: $a$ ) 'MIBG' or 'metaiodobenzylguanidine' and $b$ ) 'REM' or 'RBD'. No beginning date limit was used; the search was updated until December 12, 2010. No language restriction was used.

\section{Study selection}

Studies or subsets in studies investigating MIBG scintigraphy in patients with RBD were eligible for inclusion. Review articles, editorials or letters, comments, and case reports (studies with no more than two cases of RBD) were excluded from this review.
Two researchers (GT and FC) independently reviewed the titles and the full-text version of the retrieved articles to determine their eligibility for inclusion.

\section{Data abstraction}

For each included study, information was collected concerning basic study (author names, journal, year of publication, and country of origin), patient characteristics (mean age, sex, number of patients in whom MIBG scintigraphy was performed, and number and types of disease investigated), and MIBG scintigraphy parameters (tracer, early and delayed H/M, WR).

\section{Results \\ Literature search}

The comprehensive computer literature search from the PubMed/MEDLINE and EMBASE databases revealed 16 articles that were retrieved in full-text version. After reviewing the full text, 2 articles were excluded as review, 4,14 1 article was excluded as letter, ${ }^{15} 1$ article was excluded because MIBG scintigraphy was not performed in patients with $\mathrm{RBD},{ }^{16} 6$ articles were excluded as case reports or studies, including no more than 2 cases of RBD. ${ }^{17-22}$

Finally, 6 studies, comprising a total sample size of 139 patients with RBD, met all inclusion and exclusion criteria, and they were included in this systematic review. ${ }^{23-28}$

The characteristics of the included studies are presented in Tables 1 and 2.

\section{Literature data discussion}

In 2006, Miyamoto et al ${ }^{23}$ performed cardiac MIBG scintigraphy in 13 patients with confirmed idiopathic RBD, 12 agematched patients with $\mathrm{PD}$, and 8 age-matched controls. The authors reported a markedly reduced cardiac MIBG uptake, consistent with a sympathetic denervation, in idiopathic $\mathrm{RBD}$; they also demonstrated that this reduction is of the

Table I Basic study and patients characteristics

\begin{tabular}{|c|c|c|c|c|c|c|c|c|}
\hline Authors & Year & Country & $\begin{array}{l}\text { Number of patients } \\
\text { who performed MIBG }\end{array}$ & $\begin{array}{l}\text { Mean age } \\
\text { (years) }\end{array}$ & Male (\%) & RBD & Other LBD & Other diseases \\
\hline Miyamoto et $\mathrm{al}^{23}$ & 2006 & Japan & $25+8$ Controls & 69 & 61 & 13 & 12 PD & 0 \\
\hline Miyamoto et al ${ }^{24}$ & 2008 & Japan & $86+9$ Controls & 68 & 63 & 31 & 26 PD; 6 DLB & I0 MSA; I 3 PSP \\
\hline Miyamoto et $\mathrm{al}^{25}$ & 2009 & Japan & 63 & 64 & 81 & 47 & 0 & 16 OSAS \\
\hline Kashihara et $\mathrm{al}^{26}$ & 2010 & Japan & $235+50$ Controls & 73 & 55 & 13 & 222 PD & 0 \\
\hline Nomura et $\mathrm{al}^{27}$ & 2010 & Japan & 49 & 70 & 37 & 26 & 23 PD; no RBD & 0 \\
\hline Fujishiro et $\mathrm{al}^{28}$ & 2010 & Japan & 9 & 71 & 78 & 9 & 0 & 0 \\
\hline
\end{tabular}

Abbreviations: PD, Parkinson's disease; LBD, Lewy body disease; DLB, dementia with Lewy bodies; RBD, REM sleep behavior disorder; MSA, multiple system atrophy; PSP, progressive supranuclear palsy; OSAS, obstructive sleep apnea syndrome; MIBG, metaiodobenzylguanidine. 


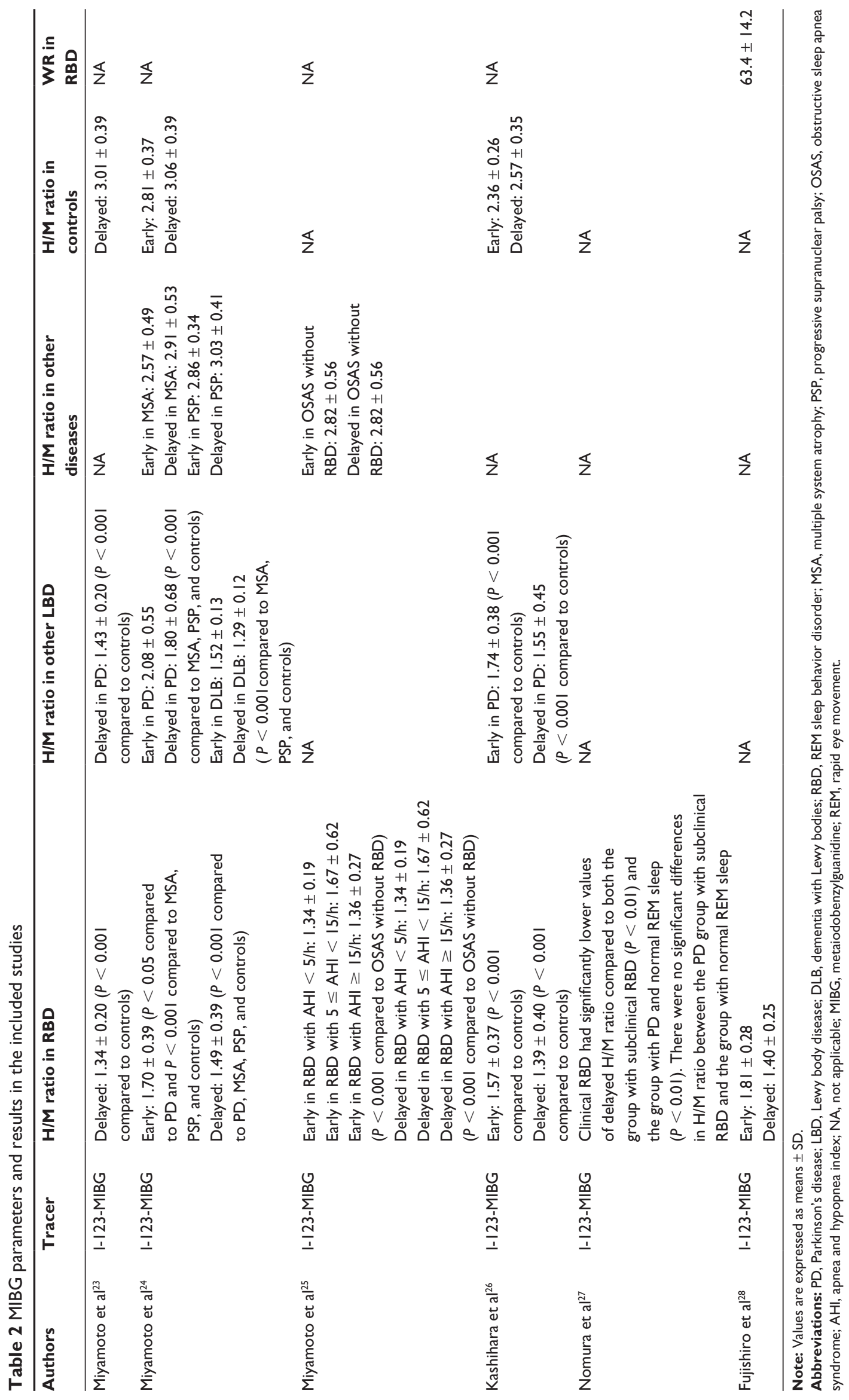


same magnitude as that found in patients with PD. The delayed $\mathrm{H} / \mathrm{M}$ ratio was reduced in patients with either idiopathic RBD or PD compared to controls (each $P<0.001$ ). The results are consistent with the hypothesis that idiopathic RBD may be an LBD.

In 2008, Miyamoto et $\mathrm{al}^{24}$ compared cardiac MIBG uptake between idiopathic RBD, PD, MSA, DLB, progressive supranuclear palsy (PSP), and control subjects. A markedly reduced cardiac MIBG uptake was detected in both the early and the delayed images in 29 of 31 (93.5\%) patients with idiopathic RBD, 21 of 26 (75\%) patients with PD, and 6 of $6(100 \%)$ patients with DLB. The cardiac MIBG uptake was normal in patients with MSA $(n=10)$, PSP $(n=13)$, and controls $(n=9)$. The early and delayed H/M ratio was significantly reduced in patients with idiopathic RBD compared to MSA, PSP, and controls ( $P<0.001$ in each subject group) and $\mathrm{PD}$ in early images $(P<0.05)$. There was a correlation between the early and delayed $\mathrm{H} / \mathrm{M}$ ratio and the duration of disease in the idiopathic RBD group (early: $r=-0.477$, $P=0.0066$; delayed: $r=-0.3578, P=0.0481)$. In conclusion, a marked reduction in cardiac MIBG uptake was found in patients with idiopathic RBD compared to control subjects and patients with clinically diagnosed PSP and MSA, but similar to those with clinically diagnosed PD and DLB.

In 2009, Miyamoto et $\mathrm{al}^{25}$ investigated whether idiopathic RBD complicated with moderate-to-severe obstructive sleep apnea could be differentiated from obstructive sleep apnea syndrome (OSAS) without RBD based on cardiac MIBG scintigraphic pattern. These authors studied 63 age-matched patients with idiopathic RBD $(n=47)$ and moderate-to-severe OSAS ( $\mathrm{n}=16)$. Cardiac MIBG uptake, based on early and delayed $\mathrm{H} / \mathrm{M}$, was significantly decreased in idiopathic RBD compared with OSAS patients $(P<0.001)$. Five of the 16 patients $(31.2 \%)$ in the OSAS group had a history of dream-enactment behavior, mimicking a RBD, but they did not have a decrease in cardiac MIBG uptake. These findings demonstrated that cardiac MIBG scintigraphy was a useful, supportive diagnostic indicator for LBD in moderate-tosevere OSAS patients.

Recently, Kashihara et $\mathrm{al}^{26}$ performed cardiac MIBG scintigraphy in patients with RBD and compared the findings with those of idiopathic PD. A total of 13 RBD, 222 PD, and 50 controls underwent MIBG scintigraphy. H/M ratios were significantly lower in patients with RBD and PD when compared to the control group $(P<0.001)$. H/M ratios were lower for delayed than for early images in patients with RBD and idiopathic Parkinson's disease (IPD) $(P<0.001)$, whereas the controls had higher $\mathrm{H} / \mathrm{M}$ ratios for delayed images $(P<0.001)$. H/M ratios did not differ significantly between patients with RBD and $\mathrm{PD}$; nevertheless, $\mathrm{H} / \mathrm{M}$ ratio were significantly lower for patients with RBD than for those with IPD at Hoehn and Yahr stages 1 and $2(P<0.001)$ and equaled those with PD at Hoehn and Yahr stages 3, 4, and 5. These findings demonstrated that: $a$ ) RBD has features of cardiac MIBG uptake reductions in common with other LBD; $b$ ) cardiac MIBG uptake was more markedly reduced in patients with RBD than in patients with early-stage PD; therefore, RBD may not necessarily be a prodromal condition of PD with respect to MIBG scintigraphic results.

Recently, Nomura et $\mathrm{al}^{27}$ have investigated the association between MIBG scintigraphic findings and RBD measures among PD patients in order to clarify whether the existence of RBD accelerates autonomic dysfunction in PD; the authors performed clinical interviews to assess RBD symptoms, polysomnogram recordings, and MIBG scintigraphy on 49 PD patients. The patients were divided into 3 groups (PD with clinical RBD, PD with subclinical RBD, and PD with normal REM sleep). PD patients with clinical RBD had reduced cardiac MIBG uptake, as determined by delayed H/M ratio, compared to those with subclinical $\mathrm{RBD}$ and those with normal REM sleep. Multiple linear regression analysis revealed that only the existence of RBD symptoms was significantly associated with reduced MIBG uptake among PD patients without dementia after adjusting for demographic and PD symptom-related variables. The authors concluded that PD patients with clinical RBD might suffer from wider $\alpha$-synuclein pathology, including reduced cardiac sympathetic ganglia function as reflected by a lowered MIBG uptake.

Recently, Fujishiro et al, ${ }^{28}$ based on the prevailing evidence that RBD often precedes the onset of DLB, compared ${ }^{18} \mathrm{~F}$-FDG PET scans of nine nondemented patients reporting recurrent nocturnal dream-enactment behavior with the normative database using three-dimensional stereotactic surface projection images. All patients underwent clinical and neuropsychological examinations as well as cardiac MIBG scintigraphy. None had dementia or met the clinical criteria for DLB or PD. Four patients were found to have diffuse areas of reduced cerebral metabolic rate of glucose (CMRglc), predominantly in the occipital lobe, which is the preferentially affected region in DLB patients. In contrast, 5 patients showed no such occipital hypometabolism; instead, these 5 patients showed hypometabolism in the left anterior cingulate gyrus, right frontal lobe, and right anterior temporal lobe, which are the preferentially affected regions in PD rather than DLB. All patients showed reduced cardiac 
MIBG uptake, consistent with underlying LBD; there were no differences in the $\mathrm{H} / \mathrm{M}$ ratio between the groups with and without diffuse occipital hypometabolism. The authors concluded that variations in ${ }^{18} \mathrm{~F}-\mathrm{FDG}$ PET scans raise the possibility that the specific pattern of CMRglc reduction may predict developing DLB in patients with idiopathic RBD.

\section{Limitations}

One possible limitation of this systematic review is that the study population in the final reviewed articles was only composed by Japanese patients. Therefore, the results might not always account for all patients with RBD, because of the racial difference. A possible explanation of these data is the wide number of Japanese studies about the application of MIBG scintigraphy in the field of neurology. ${ }^{2}$

Additional work with myocardial MIBG scintigraphy in RBD, particularly in countries outside of Japan, is clearly warranted to confirm the usefulness of this technique in this field.

All the studies included in this review used the H/M ratio to evaluate sympathetic denervation in patients with $\mathrm{RBD}$. WR is an important index, as well as $\mathrm{H} / \mathrm{M}$ ratio, in the assessment of myocardial sympathetic denervation. Nevertheless, only 1 out of 6 studies selected reported WR values in patients with RBD. A possible explanation of this finding is that considering the very low MIBG uptake in the heart in patients with RBD and other LBD, both in the early and in the delayed images, the WR from the heart is not cited. ${ }^{2}$

\section{Conclusion}

From the literature analysis, we conclude that RBD patients usually show a markedly reduced cardiac MIBG uptake, consistent with a sympathetic denervation, a typical finding of a LBD.

\section{Disclosure}

The authors report no conflicts of interest in this work.

\section{References}

1. Yamashina S, Yamazaki J. Neuronal imaging using SPECT. Eur J Nucl Med Mol Imaging. 2007;34(6):939-950.

2. Nakajima K, Yoshita M, Matsuo S, Taki J, Kinuya S. Iodine-123-MIBG sympathetic imaging in Lewy-body diseases and related movement disorders. Q J Nucl Med Mol Imaging. 2008;52(4):378-387.

3. Treglia G, Cason E, Gabellini A, Giordano A, Fagioli G. Recent developments in innervation imaging using iodine-123-metaiodobenzylgu anidine scintigraphy in Lewy body diseases. Neurol Sci. 2010;31(4): 417-422.
4. Rascol O, Schelosky L. 123I-metaiodobenzylguanidine scintigraphy in Parkinson's disease and related disorders. Mov Disord. 2009;24 Suppl 2: S732-S741.

5. Hishikawa N, Hashizume Y, Yoshida M, Sobue G. Clinical and neuropathological correlates of Lewy body disease. Acta Neuropathol. 2003;105(4):341-350.

6. Taki J, Yoshita M, Yamada M, Tonami N. Significance of 123I-MIBG scintigraphy as a pathophysiological indicator in the assessment of Parkinson's disease and related disorders: it can be a specific marker for Lewy body disease. Ann Nucl Med. 2004;18(6):453-461.

7. Orimo S, Amino T, Itoh $\mathrm{Y}$, et al. Cardiac sympathetic denervation precedes neuronal loss in the sympathetic ganglia in Lewy body disease. Acta Neuropathol. 2005;109(6):583-588.

8. Oka H, Yoshioka M, Morita M, et al. Reduced cardiac 123I-MIBG uptake reflects cardiac sympathetic dysfunction in Lewy body disease. Neurology. 2007;69(14):1460-1465.

9. Braak H, Ghebremedhin E, Rüb U, Bratzke H, Del Tredici K. Stages in the development of Parkinson's disease-related pathology. Cell Tissue Res. 2004;318(1):121-134.

10. Boeve BF. REM sleep behavior disorder: updated review of the core features, the REM sleep behavior disorder-neurodegenerative disease association, evolving concepts, controversies, and future directions. Ann N Y Acad Sci. 2010;1184:15-54.

11. Schenck CH, Bundlie SR, Mahowald MW. Delayed emergence of a parkinsonian disorder in $38 \%$ of 29 older men initially diagnosed with idiopathic rapid eye movement sleep behaviour disorder. Neurology. 1996;46(2):388-393.

12. Iranzo A, Molinuevo JL, Santamaría J, et al. Rapid-eye-movement sleep behaviour disorder as an early marker for a neurodegenerative disorder: a descriptive study. Lancet Neurol. 2006;5(7):572-577.

13. Postuma RB, Gagnon JF, Vendette M, Fantini ML, Massicotte-Marquez J, Montplaisir J. Quantifying the risk of neurodegenerative disease in idiopathic REM sleep behavior disorder. Neurology. 2009;72(15):1296-1300.

14. Postuma RB, Montplaisir J. Predicting Parkinson's disease - why, when, and how? Parkinsonism Relat Disord. 2009;15 Suppl 3:S105-S109.

15. Schenck CH, Mahowald MW. Subclinical REM sleep behavior disorder and its clinical and research implications. Sleep. 2008;31(12):1627.

16. Postuma RB, Lanfranchi PA, Blais H, Gagnon JF, Montplaisir JY. Cardiac autonomic dysfunction in idiopathic REM sleep behavior disorder. Mov Disord. 2010;25(14):2304-2310.

17. Fröhlich I, Pilloy W, Vaillant M, Diederich NJ. Myocardial MIBG scintigraphy: a useful clinical tool?: a retrospective study in 50 parkinsonian patients. Neurol Sci. 2010;31(3):403-406.

18. Miyamoto T, Miyamoto M, Iwanami M, Hirata K. Three-year follow-up on the accumulation of cardiac (123)I-MIBG scintigraphy in idiopathic REM sleep behavior disorder. Sleep Med. 2009;10(9):1066-1067.

19. Oguri T, Tachibana N, Mitake S, Kawanishi T, Fukuyama H. Decrease in myocardial 123I-MIBG radioactivity in REM sleep behavior disorder: two patients with different clinical progression. Sleep Med. 2008;9(5):583-585.

20. Minguez-Castellanos A, Chamorro CE, Escamilla-Sevilla F, et al. Do alpha-synuclein aggregates in autonomic plexuses predate Lewy body disorders?: a cohort study. Neurology. 2007;68(23):2012-2018.

21. Koyama S, Tachibana N, Masaoka Y, Homma I, Kawamura M. Decreased myocardial (123)I-MIBG uptake and impaired facial expression recognition in a patient with REM sleep behavior disorder. Mov Disord. 2007;22(5):746-747.

22. Kashihara K, Imamura T. Reduced myocardial 123I-MIBG uptake in a patient with idiopathic rapid eye movement sleep behavior disorder. Mov Disord. 2007;22(1):150-151.

23. Miyamoto T, Miyamoto M, Inoue Y, Usui Y, Suzuki K, Hirata K. Reduced cardiac 123I-MIBG scintigraphy in idiopathic REM sleep behavior disorder. Neurology. 2006;67(12):2236-2238.

24. Miyamoto T, Miyamoto M, Suzuki K, Nishibayashi M, Iwanami M, Hirata K. 123I-MIBG cardiac scintigraphy provides clues to the underlying neurodegenerative disorder in idiopathic REM sleep behavior disorder. Sleep. 2008;31(5):717-723. 
25. Miyamoto T, Miyamoto M, Suzuki K, et al. Comparison of severity of obstructive sleep apnea and degree of accumulation of cardiac 123I-MIBG radioactivity as a diagnostic marker for idiopathic REM sleep behavior disorder. Sleep Med. 2009;10(5):577-580.

26. Kashihara K, Imamura T, Shinya T. Cardiac 123I-MIBG uptake is reduced more markedly in patients with REM sleep behavior disorder than in those with early stage Parkinson's disease. Parkinsonism Relat Disord. 2010;16(4):252-255.
27. Nomura T, Inoue Y, Högl B, et al. Relationship between (123)I-MIBG scintigrams and REM sleep behavior disorder in Parkinson's disease. Parkinsonism Relat Disord. 2010;16(10):683-685.

28. Fujishiro H, Iseki E, Murayama N, et al. Diffuse occipital hypometabolism on [18 F]-FDG PET scans in patients with idiopathic REM sleep behavior disorder: prodromal dementia with Lewy bodies? Psychogeriatrics. 2010;10(3):144-152.

\section{Publish your work in this journal}

Research and Reports in Nuclear Medicine is an international, peerreviewed, open access journal publishing original research, reports, reviews and commentaries on all areas of nuclear medicine. The manuscript management system is completely online and includes a very quick and fair peer-review system. Visit http://www.dovepress.com/ testimonials.php to read real quotes from published authors. 